\section{Brightness function: Role of the pupil*}

\author{
JOSEPH C. STEVENS $†$ and LAWRENCE E. MARKS \\ John B. Pierce Foundation Laboratory and Yale University \\ New Haven, Connecticut 06519
}

Pairs of brightness functions were generated by magnitude estimation, one pair for each of three flash durations $(0.5,1.0$, and $3.0 \mathrm{sec})$. Each pair comprised a function obtained with an artificial pupil and a function obtained with the uncontrolled natural pupil. The pupil turned out to have little or no effect on the form and slope of the power functions for brightness, even when flash duration was as long as 3.0 sec.

This study attempts to clarify the practical role of the pupil in experiments designed to scale apparent brightness. In the same experimental session, a given $O$ made magnitude estimations of several levels of brightness of a target sometimes viewed through an artificial pupil and sometimes through the natural (uncontrolled) pupil. This procedure was designed to generate a pair of brightness functions that could be compared directly with respect to their constants and thereby help to elucidate the role of the pupil over a large portion of the eye's dynamic range. Since the pupil size might be expected to play a larger and larger role the longer the flash duration, duration was also varied in the experiments from 0.5 to 3.0 sec.

From experiments of Stiles and Crawford (1933), we know that pupil size influences the effective strength of cone stimulation less than was once believed. Light that enters the eye through the center of the pupil has greater luminous efficiency than that entering through the periphery. Therefore, a given percentage change in pupil area is not always equivalent to the same percentage change in luminance. It is difficult, however, to predict in a given situation and for a given $O$ the exact effect of the pupil size on the strength of stimulation.

Figure 1 shows the maximum effect that the pupil might be expected to exert on photopic brightness. The prediction is based on Le Grand's (1957, p. 104) computations of the relations (uncontrolled pupil) among target luminance, retinal illuminance, and effective retinal illuminance (i.e., retinal illuminance corrected for the Stiles-Crawford phenomenon). Assume that the apparent brightness grows as the cube root of the effective retinal illuminance (there is considerable

* This research was supported by Grant AFOSR-70-1950 with the Air Force Office of Scientific Recearch.

tPresent address: John B. Pierce Foundation Laboratory, 290 Consreas Avenue, New Haven, Connecticut 06519. evidence, stated below, that this relation is approximately correct for the dark-adapted eye and for target flashes subtending about 3-5 deg of visual arc). This cube-root function is shown as $A$ in Fig. 1, i.e., as a straight line having a slope of 0.33 in log-log coordinates.

Function B in Fig. 1 shows how the cube-root function is altered when the Stiles-Crawford phenomenon is not taken into account; it relates brightness to the retinal illuminance per se rather than to effective retinal illuminance. The alteration is relatively small and operates mainly to steepen slightly the slope of the brightness function.

Function $C$ in Fig. 1 shows how the cube-root function is altered when brightness is plotted as a function of target luminance viewed through the uncontrolled pupil; in other words, neither the changing size of the pupil nor the Stiles-Crawford effect is taken into account. The overall slope of $C$ is flatter than those of $A$ and $B$. The ratio of Slope $A$ to Slope $C$ (ignoring the slight curvature of $C$ ) is $0.33 / 0.28$.

Figure 1 predicts, therefore, that the main effect of an uncontrolled pupil would be to yield an exponent about $15 \%$ smaller than that obtained with the use of a pupil of fixed area. Actually, Fig. 1 exaggerates the predicted difference. For one thing, Le Grand's calculations of pupil size are based on steady-state measurement, i.e., the pupil is allowed to reach minimal size in response to a given level of stimulation. But the brightness function is typically measured with transient (1 or $2 \mathrm{sec}$ ) stimulation of the dark-adapted eye (pupil dilated). Under this condition, the uncontrolled pupil could not possibly attain minimal size before the termination of stimulation. For another thing, at the onset of stimulation, the dark-adapted retina receives the maximal level of illumination; this level is continuously attenuated after the onset of pupil constriction, but it is questionable if this attenuation actually affects the brightness of relatively brief flashes. The impression of brightness may already be formed in the early stages

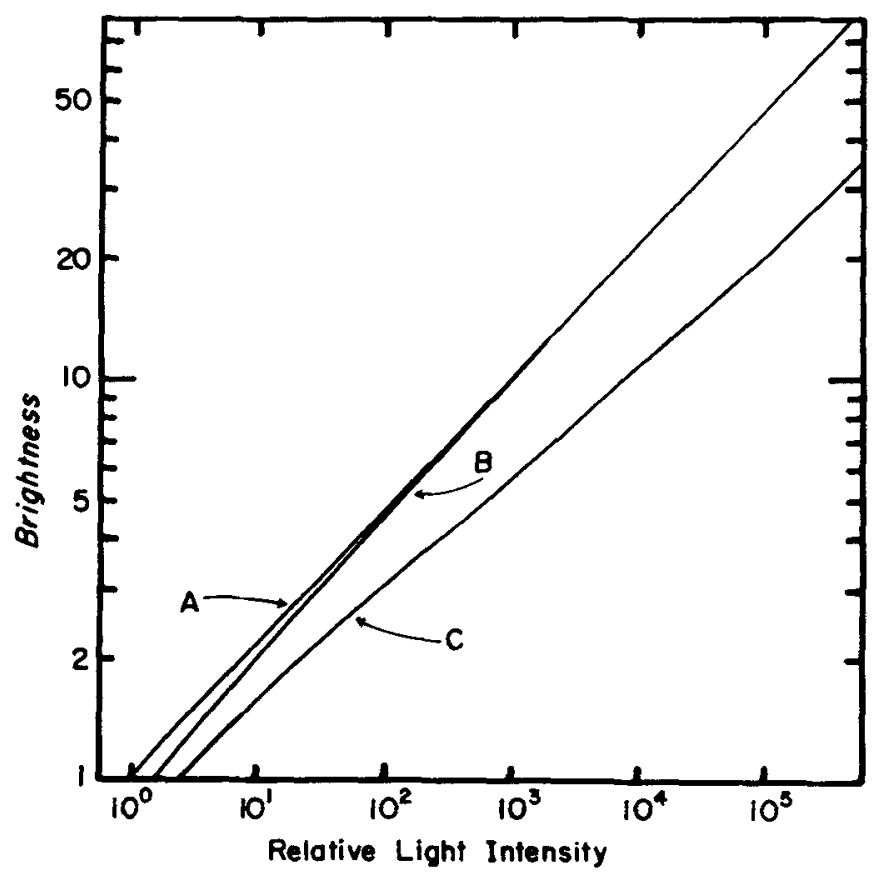

Fig. 1. Showing the predicted maximum effect of the pupil on the brightness function. Line $A$ is a cube-root function relating brightneas and effective retinal illuminance (both coordinates are logarithmic). Line B shows how $A$ is altered if the Stiles-Crawford effect is not taken into account. Line $C$ shows how $A$ is altered if both pupil size and Stiles-Crawford effect are not taken into account. 


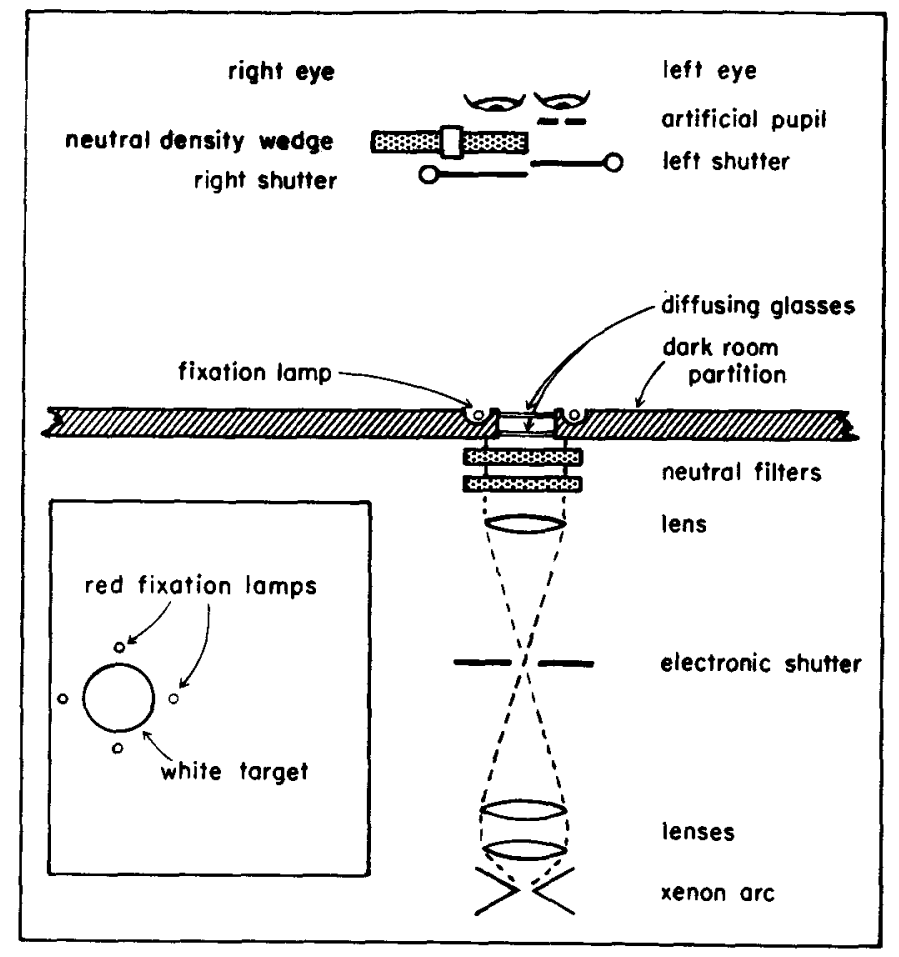

Fig. 2. Schematic diagram of the apparatus.

of stimulation. Considering the rapid diminution of pupil size (Page, 1941) and of retinal sensitivity due to light adaptation (Wallace, 1937), it is amazing how stable the perceived brightness is. Under certain conditions, one can, of course, observe a decline in brightness under continuous stimulation at a constant level; the decline is, however, about equally noticeable through an artificial and an uncontrolled pupil and must, therefore, be mediated primarily by light adaptation.

These considerations suggest that the pupil's effect on a given visual task can best be gauged by experiment. One might ask, why bother since the effects of the pupil can usually be controlled with an artificial pupil, with drugs, or with the Maxwellian view. One reason is that people who have a practical concern with visual measurement (such as illumination engineers) are interested in vision mainly as it takes place through the natural pupil. Another reason is the practical advantage of knowing the conditions under which the artificial pupil or equivalent control can be dispensed with in the interest of optical simplicity and gain in effective luminance.

\section{APPARATUS}

As shown in Fig. 2, the flux from a 1,000-W xenon lamp (Hanovia $976 \mathrm{C}-1$ ) transilluminated a pair of diffusing glasses set in a hole in a partition of a dark booth. The visual target thereby formed was circular (diam $=2.75 \mathrm{~cm}$, or $3.3 \mathrm{deg}$ at viewing distance of $47 \mathrm{~cm}$ ), white (flat spectrum), uniform, and relatively nondirectional. Its luminance was controlled with neutral density filters and its duration with a fast electronic shutter (Gerbrands Model G11165).

Secondary shutters near the $O$ determined which eye would view the target on a given trial. In front of the left eye was an artificial pupil (diam $=2.5 \mathrm{~mm}$ ). In front of the right eye was a neutral density wedge set to attenuate the target by $6 \mathrm{~dB}$. Under this condition, the brightness of the target appeared approximately the same to the two eyes. Luminance was measured with a Gamma Scientific (Model 2000) telephotometer.

Surrounding the target were four small red fixation lights (see Fig. 1 for arrangement). These lights went out whenever the target came on.

\section{PROCEDURE}

Each of 13 male Os served in two virtually identical sessions spaced at least a day apart. A session began with $10 \mathrm{~min}$ of dark adaptation using red goggles, plus a minute or two in the dark. The task was magnitude estimation of brightness (no designated modulus). The instruction was to assign numbers in proportion to the apparent brightness experienced at the end of a flash. It was explained that the flashes would vary in duration as well as in brightness but that duration should be disregarded in forming the judgment of terminal brightness. A few practice judgments were made (without feedback) at the start. Then the $O$ made 48 magnitude estimations ( 8 luminance levels by 3 durations by 2 eyes). The levels ranged from 53 to $108 \mathrm{~dB}$ re $1 / \pi \cdot 10^{-6}$ candela $/ \mathrm{m}^{2}$ (left eye) and from 47 to $102 \mathrm{~dB}$ (right eye); the durations were $0.5,1.0$, and 3.0 sec.

The stimulus order was different in every experimental session and was irregular with respect to luminance level and duration. The eye stimulated alternated from one trial to the next. The wait between trials was about 20 sec, so that an eye was in the dark approximately $40 \mathrm{sec}$ between stimulations.

\section{RESULTS}

The estimations of each of the 48 stimuli were averaged geometrically across the 26 sessions of the 13 Os and the geometric means plotted in the log-log coordinates of Fig. 3. The

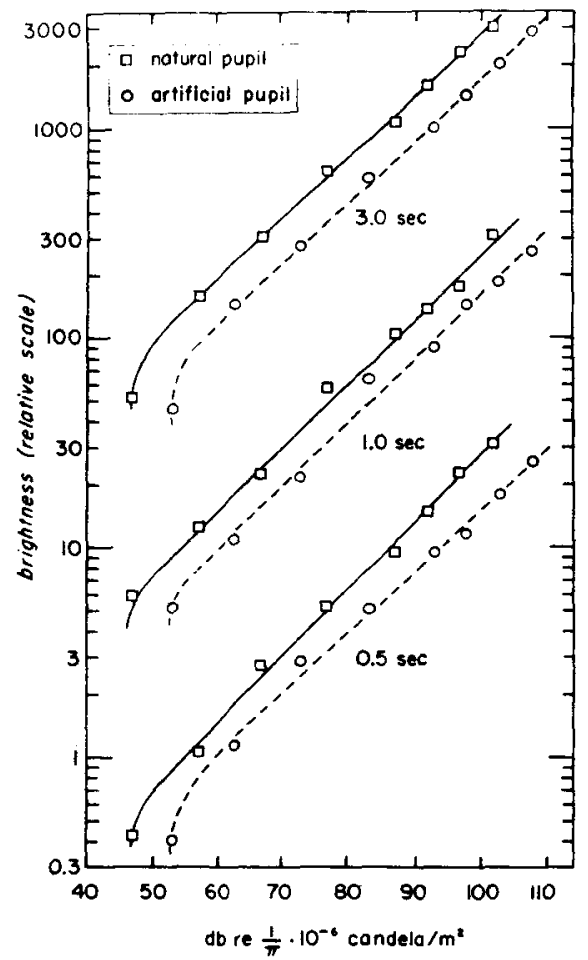

Fig. 3. Three pairs of brightness functions in $\log -\log$ coordinates. For clarity, the pairs have been arbitrarily separated from each other in the vertical dimension. To do this, the brightness estimates for $3.0 \mathrm{sec}$ were multiplied by 100 , those for $1.0 \mathrm{sec}$ by 10 , and those for $0.5 \mathrm{sec}$ by 1.0 . 
Table 1

Constants of the Brightness Equation $\psi=k\left(\varphi-\varphi_{0}\right)^{\beta}$

\begin{tabular}{lccccc}
\hline & Seconds & $\beta$ & log $k$ & $\varphi_{\text {o }}$ in db & r \\
\hline \multirow{3}{*}{ Artificial Pupil } & 0.5 & 0.29 & -1.70 & 52 & 0.998 \\
& 1.0 & 0.30 & -1.82 & 49 & 0.996 \\
Natural Pupil & 3.0 & 0.29 & -1.64 & 52 & $0.999+$ \\
& 0.5 & 0.32 & -1.72 & 45 & 0.999 \\
& 1.0 & 0.30 & -1.59 & 42 & 0.999 \\
\hline
\end{tabular}

Note-r is the Pearson coefficient of correlation

curves in Fig. 3 have the power formula:

$$
\log \psi=\beta \log \left(\varphi-\varphi_{0}\right)+\log k
$$

or in nonlogarithmic form:

$$
\psi=\mathbf{k}\left(\varphi-\varphi_{\mathrm{o}}\right)^{\beta}
$$

A power formula has been found by numerous investigators of brightness (e.g., Hopkinson, 1956; Ekman, Eisler, \& Künnapas, 1960; Onley, 1961; J. C. Stevens \& S. S. Stevens, 1963; Jameson, 1965; Root \& Ross, 1965; Marks, 1966). Like many other sensory continua, brightness conforms to the psychophysical power law proposed by S. S. Stevens (1957).

In Table 1 are listed the values of the constants $\beta, k$, and $\varphi_{0}$ computed separately for the six brightness functions ( 3 durations by 2 conditions of viewing). These constants were computed with an iterative least-squares method (see J. C. Stevens, 1967). The Pearson coefficient of correlation ( $r$ ) in Table 1 reflects the strength of the linear relation between $\log \psi$ and $\log$ $\left(\varphi-\varphi_{0}\right)$.

It is apparent from Fig. 3 and Table 1 that the overall form and slope of the brightness functions were influenced little by the pupil. The position of the function depends, of course, on the pupil's size, i.e., on the amount of light admitted and on the operation of the Stiles-Crawford effect. That is why a pair of functions in Fig. 3 is separated by a nearly constant horizontal distance.

For target durations of 1.0 and $3.0 \mathrm{sec}$, the two pupillary conditions produced functions having virtually identical slopes, but for the 0.5 -sec duration the artificial pupil yielded a noticeably flatter function than did the uncontrolled pupil. This difference may be due to chance; it is difficult to explain in terms of the pupil because Fig. 1 predicts that, if anything, the function obtained with the artificial pupil should be steeper, not flatter, than that obtained with the uncontrolled pupil. Moreover, any effect of changing pupil size ought to grow, not diminish, with increasing target duration.

The hypothesis that the pupil might noticeably influence the slope of the brightness function when target duration lasted as long as $3.0 \mathrm{sec}$ received no support from the experimental results. There are several possible reasons for this outcome. One might be that brightness actually did change as the pupil constricted, but the Os, for some reason, felt compelled to ignore the brightness changes and to judge the maximal brightness perceived (contrary to the instructions). An alternative is that the eye cannot readily detect the relatively slow changes in retinal illuminance such as those produced by pupillary contraction, even though the eye easily detects smaller changes when they are abrupt. The visual system may in some way suppress the effectiveness of the attenuation of intensity of stimulation caused by pupillary constriction. In line with this alternative is the finding that the detectability of sinusoidal modulations of luminance continues to decrease as modulation frequency decreases to very low rates, e.g., $0.1 \mathrm{~Hz}$ (Keesey, 1970). The possibility of suppression of a temporal gradient is reminiscent of and perhaps analogous to the eye's tendency to discount spatial gradients of illumination unless the gradients are discontinuous or precipitous (Békésy, 1960; Ratliff, 1965).

Under steady-state conditions, the pupil may influence brightness much more than it does the relatively brief flashes employed here. However, in some experiments that involve transient stimulation of the dark-adapted eye, such as the typical brightness scaling experiment, it would seem unnecessary to control the pupil size.

\section{REFERENCES}

BÉKESY, G. von. Neural inhibitory units of the eye and skin. Quantitative description of contrast phenomena. Journal of the Optical Society of America, 1960, 50, 1060-1070

EKMAN, G., EISLER, H., \& KÜNNAPAS, $T$. Brightness scales for monochromatic light. Scandanavian Journal of Psychology, 1960, 1, 41-48.

HOPKINSON, R. G. Light energy and brightness sensation. Nature, 1956, 178, 1065-1066.

JAMESON, D. Threshold and suprathreshold relations in vision. Proceedings of the International Colour Meeting (Lucerne), 1965, 1, 128-136.

KEESEY, U. T. Variables determining flicker sensitivity in small fields. Journal of the Optical Society of America, 1970, $60,390-398$.

Le GRAND, Y. Light, colour and vision. London: Chapman \& Hall, 1957.

MARKS, L. E. Brightness as a function of retinal locus. Perception \& Psychophysics, 1966, 1, 335-341.

ONLEY, J. W. Light adaptation and the brightness of brief foveal stimuli. Journal of the Optical Society of America, 1961, $51,667-673$.

PAGE, $H_{n}$ E. The relation between area of stimulation and intensity of light at various levels of visual excitation as measured by pupil constriction. Journal of Experimental Psychology, 1941, 29, 177-200.

RATLIFF, F. Mach bands: Quantitative studies on neural networks in the retina. San Francisco: Holden-Day, 1965.

ROOT, R. T., \& ROSS, S. Further validation of subjective scales for loudness and brightness by means of cross-modality matching. American Journal of Psychology, 1965, 78, 285-289.

STEVENS, J. C. Brightness function: Binocular versus monocular stimulation. Perception \& Psychophysics, 1967, 2, 451-454.

STEVENS, J. C., \& STEVENS, S. S. Brightness function: Effects of adaptation. Journal of the Optical Society of America, 1963, 53, 375-385. STEVENS, S. S. On the psychophysical law. Psychological Review, 1957, 64, 153-181. STILES, W. S. \& CRAWFORD. B. H. The luminous efficiency of rays entering the eye pupil at different points. Proceedings of the Royal Society (London), 1933, $112 B, 428-450$.

WALLACE, S. R., JR. Studies in binocular interdependence: $I$. Binocular relations in macular adaptation. Journal of General Psychology, 1937, 17, 307-322.

(Received for publication July 13, 1971.) 